\title{
Measurement and compensation of residual group delay in a multi-core fiber for lensless endoscopy
}

\author{
Esben Ravn Andresen, ${ }^{1,3}$ Siddharth Sivankutty, ${ }^{1}$ Géraud Bouwmans, ${ }^{2}$ Laurent Gallais, ${ }^{1}$ \\ Serge Monneret, ${ }^{1}$ and Hervé Rigneault ${ }^{1, *}$ \\ ${ }^{1}$ Aix-Marseille Université, CNRS, Centrale Marseille, Institut Fresnel UMR 7249, 13013 Marseille, France \\ ${ }^{2} P$ hLAM CNRS, IRCICA, Université Lille 1, 59658 Villeneuve d'Ascq Cedex, France \\ ${ }^{3}$ e-mail: esben.andresen@fresnel.fr \\ *Corresponding author: herve.rigneault@fresnel.fr
}

Received 25 March 2015; revised 4 May 2015; accepted 5 May 2015; posted 5 May 2015 (Doc. ID 236511); published 26 May 2015

\begin{abstract}
We present a new experimental concept suitable for partially compensating the inter-core group delay dispersion in a multi-core fiber (MCF). First we map out the group delays of all 169 single-mode cores of a MCF using phase-shifting spectral interferometry and find the group delays distributed with standard deviation $123 \mathrm{fs}$ in a $30 \mathrm{~cm}$ long MCF. We then detail and apply the compensation scheme based on two wavefront shapers with which we narrow the group delay distribution to $65 \mathrm{fs}$. These results are relevant for lensless endoscopes employing femtosecond excitation, and we quantify the performance gain in a lensless endoscope with $\mathbf{1 5 0} \mathrm{fs}$ laser pulses as excitation and discuss possible generalizations of the concept. () 2015 Optical Society of America
\end{abstract}

OCIS codes: (060.2430) Fibers, single-mode; (170.2150) Endoscopic imaging; (180.2520) Fluorescence microscopy; (180.4315) Nonlinear microscopy; (180.5810) Scanning microscopy; (230.6120) Spatial light modulators.

http://dx.doi.org/10.1364/JOSAB.32.001221

\section{INTRODUCTION}

Recent advances in instrumentation for wavefront control such as two-dimensional spatial light modulators (2D-SLMs) and deformable mirrors (DMs) have made it possible to unscramble the modes of light that traverse a complex medium [1], essentially enabling the transmission of spatial information through said complex medium. Salient examples are the so-called lensless endoscopes, which in effect achieve imaging through a multi-mode waveguide [2-5]. In these references, the common factor is that a multi-mode fiber (MMF) was employed as the waveguide. The properties of MMF have repercussions; in particular, the mode dispersion in MMF dictates that a long coherence length light source must be used, although delivery of 520 fs pulses through MMF has recently been demonstrated though at the expense of spatial degrees of freedom [6]. A different approach to lensless endoscopes is to employ not a MMF, but a multi-core fiber (MCF), as the waveguide [7-9]. Provided the cores are single-mode and exhibit negligible cross-talk, constraints on light source coherence length are relaxed to the point that a femtosecond light source can be used, as we have shown [9]. However, the relaxation of constraints is not complete as it turns out that the group delay of individual cores is not identical. We shall term this residual differential group delay "inter-core group delay dispersion (GDD)". It can be thought of as the MCF's counterpart to the MMF's mode dispersion. Inter-core GDD (where spatial modes localized to individual cores experience different group delays) is different from the well-known chromatic dispersion (where different colors experience different group delays), and the two should not be confused.

Inter-core GDD is a significant obstacle in the realization of lensless endoscopes for nonlinear imaging. The origin of intercore GDD may be broadly attributed to (1) intrinsic contributions stemming from material and waveguide dispersion and (2) extrinsic contributions that arise from bending or twisting the MCF. A compensation of both of these factors has remained a challenge to the research community. Inter-core GDD was not treated in our latest contribution [9]. In this work, we aim to address the measurement and compensation of the inter-core GDD that arises from the intrinsic contributions. Here, we detail a method based on phase-shifting spectral interferometry for directly measuring the group delay incurred by a femtosecond pulse in each core of a MCF with 169 cores. We then detail and demonstrate a scheme for compensation of inter-core GDD and show the resulting performance gain. We note that a recent paper reports the successful measurement of the inter-core dispersion of propagation constants in a 37-core MCF with strong coupling between cores [10]. Here, we examine the subject in a MCF with uncoupled cores. 


\section{EXPERIMENT}

\section{A. Multi-Core Fiber}

Figure 1(a) shows an electron micrograph of the double-clad silica MCF with $N=169$ cores used in this study and that is identical to the one we used in [9]. It was fabricated by the stack and draw process [11]. First, Ge-doped rods drawn from a preform (Prysmian Group, parabolic refractive index profile, maximum difference of $31 \cdot 10^{-3}$ compared to silica) were inserted into a stack of 169 capillaries. This "double-stacking" step was required in order to achieve very low coupling between the single-mode cores of the final MCF. This multi-core preform was then drawn into several all-solid canes. One of these canes was put in a jacketing tube containing the air-clad structure (made by stacking 61 hollow capillaries between two tubes) and drawn to get a MCF with the following parameters: pitch, $11.8 \mu \mathrm{m}$; mode field diameter of each core, $3.6 \mu \mathrm{m}$; diameter of multi-mode inner cladding, $250 \mu \mathrm{m}$; numerical aperture (NA) of multi-mode inner cladding, 0.65 ; total outer diameter, $360 \mu \mathrm{m}$. The cross-talk was extremely low, and the measured coupling from one core to its nearest neighbor in a $30 \mathrm{~cm}$ long section was less than $-25 \mathrm{~dB}$. Figure $\underline{1(\mathrm{~b})}$ shows the convention for core indices $i$ used in the following.

\section{B. Setup}

Figure 2 shows a sketch of the experimental setup. The pulses from a $\mathrm{Yb}$ femtosecond laser (Amplitude Systèmes t-Pulse, $1035 \mathrm{~nm}, 150 \mathrm{fs} ; 50 \mathrm{MHz}, 1.3 \mathrm{~W}$, horizontally polarized) pass through magnifying telescopes (not shown) so that the collimated beam overfills the aperture of the DM (IRIS-AO, PTT-489, 163 segments), each segment of which has a piston, a tip, and a tilt degree of freedom [12]. With the piston of segment $i$, a phase can be imposed on the light impinging on that segment, $\phi_{i}^{\text {proximal }}=2 \cdot 2 \pi \cdot$ piston $/ \lambda$. Similarly, tip and tilt impose transverse $k$ vectors $\Delta k_{x}^{(i)}=2 \cdot 2 \pi / \lambda$. $\sin \left(\right.$ tip) and $\Delta k_{y}^{(i)}=2 \cdot 2 \pi / \lambda \cdot \sin ($ tilt). The Fourier plane of the DM is in the plane $\Sigma_{1}$. The DM is imaged $\left(f_{1}, f_{2}\right)$ onto a 2D-SLM (Hamamatsu X8267, $768 \times 768$ pixels, $20 \mathrm{~mm} \times 20 \mathrm{~mm}$ ). On the 2D-SLM a phase mask is inscribed that consists of $N=169$ hexagonal segments of chirped gratings on a triangular grid. Each hexagonal DM segment is imaged onto a hexagonal segment on the 2D-SLM. The phase mask $\Phi_{2 \mathrm{D}-\mathrm{SLM}}^{(i)}$ of segment $i$ centered on $\left(X_{i}, Y_{i}\right)$ on the $2 \mathrm{D}$-SLM is of the form
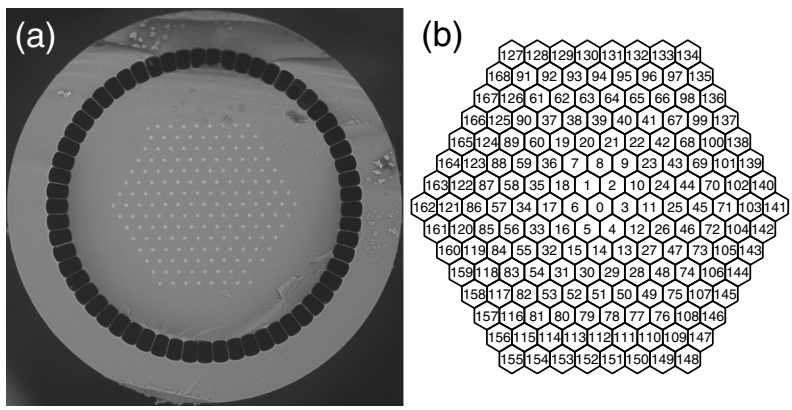

Fig. 1. Multi-core fiber with 169 single-mode cores and multimode inner cladding. (a) Electron micrograph of the multi-core fiber. (b) Core numbering convention used in the text.

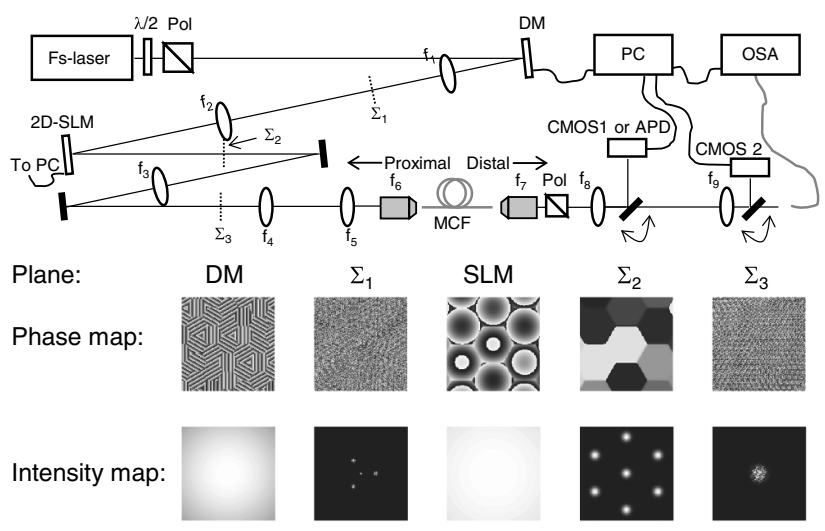

Fig. 2. Sketch of the experimental setup. Fs-laser, femtosecond laser; $\lambda / 2$, half-wave plate; Pol, polarizer; 2D-SLM, two-dimensional spatial light modulator; ID, iris diaphragm; CMOS, CMOS camera; OSA, optical spectrum analyzer; PC, personal computer; DM, deformable mirror; APD, avalanche photodiode. $f_{1}=150 \mathrm{~mm}$, $f_{2}=300 \mathrm{~mm}, f_{3}=500 \mathrm{~mm}, f_{4}=80 \mathrm{~mm}, f_{5}=75 \mathrm{~mm}, f_{6}=$ $4.51 \mathrm{~mm}, f_{7}, 20 \times$ microscope objective, $f_{8}=150 \mathrm{~mm}$, and $f_{9}=200 \mathrm{~mm}$. All lenses are in the $4-f$ geometry unless otherwise noted. The insets show sketches of phase maps and intensity maps of the laser beam in the different planes. The DM can impose three possible transverse $k$ vectors on the beamlets; this leads to three spots in $\Sigma_{1}$ (Fourier plane of DM, the spots are destined to traverse different zones of the compensation plate). The SLM (image plane of DM) can impose three possible transverse $k$ vectors on the beamlets so that all beamlets are diffracted parallel to one another; in addition, a wavefront curvature is imposed on each beamlet, which leads to an array of spots in $\Sigma_{2}$ (which will be imaged onto the MCF) and a single spot in $\Sigma_{3}$ (Fourier plane of $\Sigma_{2}$ ) containing all the beamlets.

$$
\begin{aligned}
\Phi_{2 \mathrm{D}-\mathrm{SLM}}^{(i)}(X, Y)= & \text { sawtooth }\left\{\frac{f_{1}}{f_{2}} \Delta k_{x}^{(i)} X+\frac{f_{1}}{f_{2}} \Delta k_{y}^{(i)} Y\right. \\
& \left.+\frac{\pi}{\lambda f_{\text {conc }}}\left[\left(X-X_{i}\right)^{2}+\left(Y-Y_{i}\right)^{2}\right]\right\} .
\end{aligned}
$$

Here $\Delta k_{x}^{(i)}$ and $\Delta k_{y}^{(i)}$ are the same as those introduced previously, and the $f_{1} / f_{2}$ factor takes into account the magnification from DM to 2D-SLM. The function of the 2D-SLM mask can be understood as follows. The first and second arguments of the sawtooth function give rise to a grating blazed in the direction of the incoming beamlet and with period that gives a diffracted beamlet with transverse $k$ vector equal to zero. Here, in general, the norm of $\Delta \vec{k}^{(i)}$ was either 0 or $0.0262 \mu \mathrm{m}^{-1}$ corresponding to a period of 20 pixels on the 2D-SLM. Note that in this configuration of DM and 2DSLM, all beamlets of equal $\Delta \vec{k}$ meet at the same point in the plane $\Sigma_{1}$. In addition all beamlets, whatever their $\Delta \vec{k}$, are diffracted from the 2D-SLM parallel to one another, and so they all pass through the same point in the plane $\Sigma_{3}$. Put differently, we employ the DM's tip/tilt degree of freedom to divide the beamlets into three subgroups, each destined to traverse a certain zone of a compensation plate. The setup can be dynamic - thanks to the tip/tilt degree of freedom the members of the subgroups can be interchanged on the fly. Figure $\underline{3}$ shows a schematic view of this concept. The last term in the argument of the sawtooth is responsible for setting up a 


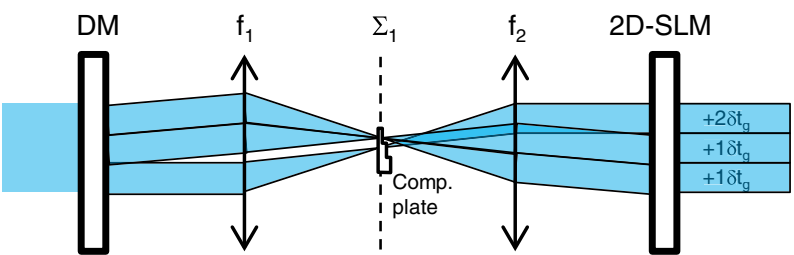

Fig. 3. Schematic view of the concept for compensating inter-core GDD. Annotations as in Fig. 2 .

concave mirror in the first diffracted order with focal length $f_{\text {conc }}=300 \mathrm{~mm}$. Each segment $i$ will then diffract a convergent beamlet into the first order. In the first order, the entire 2D-SLM thus acts as an array of concave mirrors producing a hexagonal array of spots in the plane $\Sigma_{2}$. The diffraction efficiency into the first order is around $25 \%$. The plane $\Sigma_{2}$ is imaged $\left(f_{3}, f_{4}, f_{5}, f_{6}\right)$ onto the proximal endface of the MCF. A CMOS camera is conjugated to either the bundle distal endface $\left(f_{7}, f_{8}\right.$, "CMOS1" in Fig. 2$)$ or its Fourier plane $\left(f_{7}, f_{8}\right.$, $f_{9}$, "CMOS2" in Fig. 2$)$. The probe $(\varnothing 62.5 \mu \mathrm{m}, \mathrm{MMF})$ of a fiber-coupled optical spectrum analyzer (OSA) (ANDO AQ6315A) is also placed in the Fourier plane for phase-shifting spectral interferometry [13] to measure the group delay $\Delta t_{g}^{(i)}$ of each core (see Appendix $\underline{\text { A}}$ ).

\section{Compensation Plates}

We machined two sets of glass plates with one or more holes that together acted as the group delay compensating element. The first set, sketched in Fig. 4(a), consisted of three plates: the first two were $100 \mu \mathrm{m}$ thick microscope coverslips (Menzel Gläser) with one and two holes, respectively. A pass through one coverslip corresponds to an additional group delay of $170 \mathrm{fs}$. The third was an opaque plate (a microscope coverslip covered with aluminum foil) with three holes acting as a beam block. The second set was identical apart from the fact that the coverslips were $170 \mu \mathrm{m}$ thick corresponding to a delay of $300 \mathrm{fs}$. The $\approx \varnothing 700 \mu \mathrm{m}$ holes were drilled in the coverslips by laser ablation (using an Amplitude Systèmes S-Pulse HP laser). We used galvanometric mirrors and an $\mathrm{f}$-theta lens to (a)

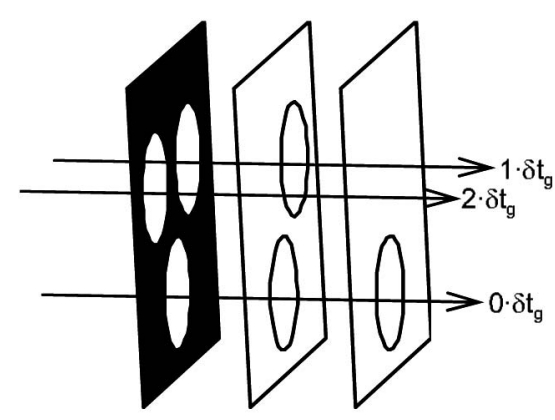

(b)

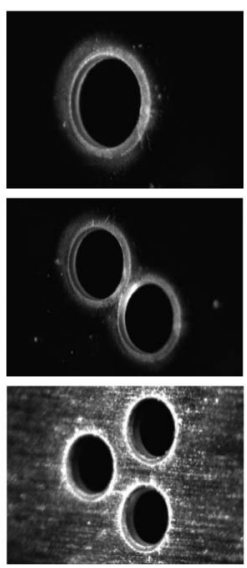

Fig. 4. (a) Sketch of the individual plates making up a compensation plate showing the alignment of holes. The compensation plate is located in the plane $\Sigma_{1}$. (b) Microscope images of each constituent plate. drill the samples along circular drilling patterns with the following irradiation parameters: beam diameter $120 \mu \mathrm{m}$, repetition rate $1 \mathrm{kHz}$, and pulse energy $0.5 \mathrm{~mJ}$. The time to drill one hole was $5 \mathrm{~s}$. Figure $4(\mathrm{~b})$ shows microscope images of the machined coverslips. The final compensation plates were obtained by sandwiching the individual plates together separated by a $200 \mu \mathrm{m}$ spacer. For compensation of inter-core GDD the compensation plate was placed in the plane $\Sigma_{1}$ (see the inset in Fig. $\underline{2}$ as well as Fig. $\underline{3}$ ), allowing us to impose one of three additional group delays on a beamlet according to its $\Delta \vec{k}$ as sketched in Fig. 4(a). We would like to point out that the parameters of the compensation plate depend only on the resolution and the range of the desired compensation, and not the number of cores of the MCF. Hence, these plates could be scaled up for any number of cores.

\section{CALCULATION OF GROUP DELAY EFFECTS}

We start out by analyzing the impact of inter-core GDD by performing analytical calculations with relevant parameters. These calculations will be a guide to the experiment.

We first quantify the effect of inter-core GDD on the duration FWHM and the peak intensity in the reconstructed focus at the distal end of the MCF as well as the two-photon excited fluorescence (2PEF) generated in the focus. The focus field $E_{\text {distal }}$ is defined as the coherent superposition of all the $N$ beamlets emanating from the $N$ cores. For simplicity we assume that all beamlets are identically equal to $\mathcal{E}$, differing only in their group delay $\Delta t_{g}^{(i)}$. We assume that all the beamlets are in phase, giving

$$
E_{\text {distal }}(t)=\sum_{i=1}^{N} \mathcal{E}\left(t-\Delta t_{g}^{(i)}\right) .
$$

For now, $\left\{\Delta t_{g}^{(i)}\right\}$ is assumed to be normally distributed with standard deviation $\sigma$. For a given $\sigma$, we generate 100 random $\left\{\Delta t_{g}^{(i)}\right\}$, and an average distal field $\left\langle E_{\text {distal }}\right\rangle$ is calculated from Eq. (2). The three curves in Fig. 5 then come about as follows: FWHM, the FWHM of $\left|\left\langle E_{\text {distal }}\right\rangle\right|^{2} ; \max (\mathrm{I})$, the maximum of $\left|\left\langle E_{\text {distal }}\right\rangle\right|^{2}$; 2PEF sig., $\int\left|\left\langle E_{\text {distal }}\right\rangle\right|^{4} \mathrm{~d} t$. From Fig. $\underline{5}$ it can be seen that all the parameters, duration FWHM, peak intensity, and generated 2PEF signal, deteriorate rapidly with increasing $\sigma$. Particularly, the generated 2PEF signal decreases by a factor of 7.5 (to 0.134 ) when $\sigma$ equals the pulse duration FWHM.

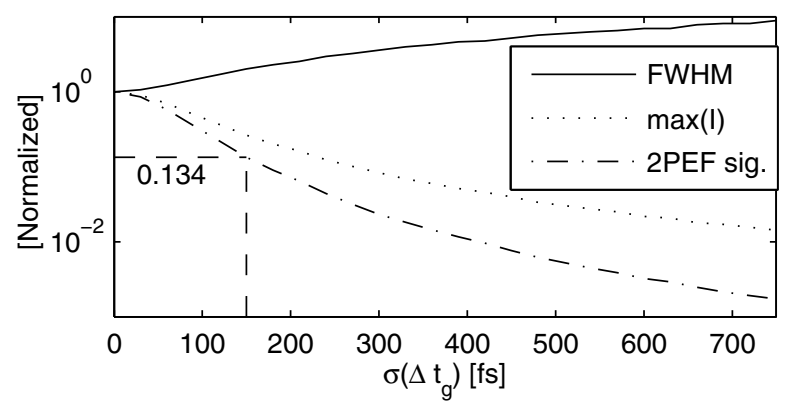

Fig. 5. Calculated mean parameters of an uncompensated pulse that has traversed a MCF in which the group delays are normally distributed with a standard deviation $\sigma$. Assumed pulse duration $\mathrm{FWHM}=150 \mathrm{fs}$. See the text for details. 


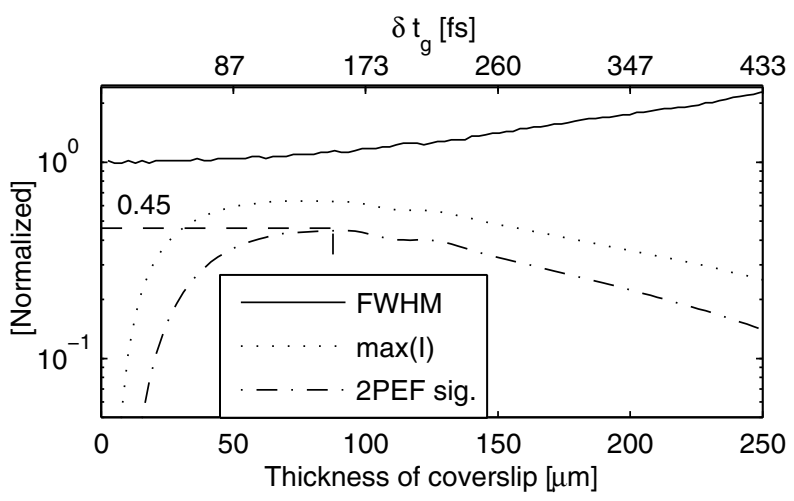

Fig. 6. Calculated pulse parameters of a compensated (after the scheme described in the text) pulse that has traversed a MCF in which the group delays are normally distributed with standard deviation $\sigma=150$ fs. See the text for details.

We now calculate the effects of the envisioned compensation scheme. The scheme allows us to impose additional group delay on arbitrary beamlet $i$ in units of $\delta t_{g}$ [see Fig. 4(a)]. The compensated group delays $\Delta t_{\text {comp, } g}^{(i)}$ are then

$$
\Delta t_{\text {comp }, g}^{(i)}=\left[\Delta t_{g}^{(i)}-\min \left(\Delta t_{g}^{(i)}\right)\right] \bmod \delta t_{g} .
$$

And the compensated distal field is

$$
E_{\text {distal }}(t)=\sum_{i=1}^{N} 0.96^{\left[\left[\Delta t_{g}^{(i)}-\min \left(\Delta t_{g}^{(i)}\right)\right] / \delta t_{g}\right\rfloor} \mathcal{E}\left(t-\Delta t_{\text {comp }, g}^{(i)}\right) .
$$

Here, $\lfloor\ldots\rfloor$ denotes rounding down to the nearest integer. The " 0.96 " term is there to emulate the reflection losses present if each $\delta t_{g}$ were to be applied by a pass through a glass plate. The outcome of this compensation process gives Fig. $\underline{6}$. This compensation scheme is seen to give a significant improvement over the uncompensated case (the curves asymptotically approach the uncompensated case going to the right). In particular, the 2PEF signal increases by a factor of 3.4 (from 0.134 in Fig. 5 to 0.45 in Fig. 6) for a glass plate thickness of $90 \mu \mathrm{m}$ corresponding to $\delta t_{g}=156$ fs.

\section{RESULTS}

\section{A. Measurement of Group Delays}

Using phase-shifting spectral interferometry (see Appendix A) we measure $\left\{\Delta t_{g}^{(i)}\right\}$ in a $30 \mathrm{~cm}$ long MCF suspended at points $28 \mathrm{~cm}$ apart. The MCF has no twist even though it was not held completely straight. So, all cores have the same geometry, and no contribution of deformation to inter-core GDD is expected-only the intrinsic contributions of material and waveguide dispersion are expected. The outcome of the measurement is presented in Fig. $\underline{7(\mathrm{a})}$ as $\Delta t_{g}^{(i)}$ versus core index $i$. Figure $7(\mathrm{~b})$ presents the histogram of the data set. The distribution has a standard deviation of $\sigma=123 \mathrm{fs}$ and resembles a normal distribution. We have not found any correlation between the $\Delta t_{g}^{(i)}$ of a core and any parameter of that core. However, in the MCF fabrication the Ge-doped rods were stacked in no particular order compared to their position in the original preform, so slow longitudinal variations in the preform could be responsible. Additionally, the double-stacking
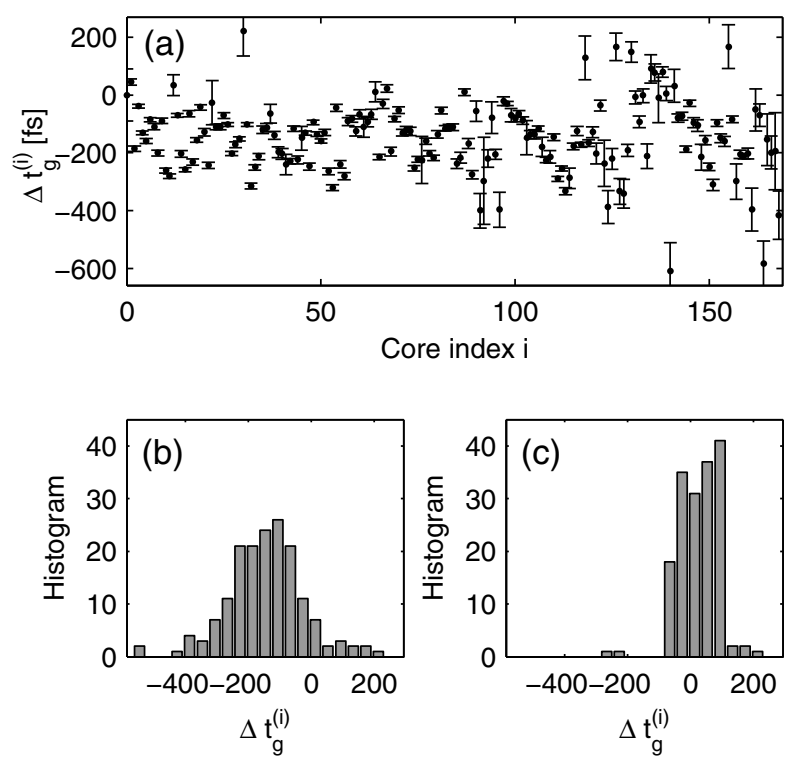

Fig. 7. Measured inter-core group delay in $30 \mathrm{~cm}$ MCF. (a) Measured group delay $\Delta t_{g}^{(i)}$ of core $i$ relative to $i=0$. $0 \leq i \leq 168$. Note that the error bars, three standard deviations, refer to the measurement uncertainty of a single delay measurement. (b) Histogram of the measured $\Delta t_{g}^{(i)}$ with 21 bins of $40 \mathrm{fs}$ width. The standard deviation, obtained from a Gaussian fit to the histogram, is $123 \mathrm{fs}$. (c) Calculated histogram with the group delay compensation scheme using $\delta t_{g}=170 \mathrm{fs}$; the standard deviation is $65 \mathrm{fs}$.

step could be responsible for introducing slight random deformations that lead to inter-core GDD. While $\sigma$ is on the order of the laser pulse duration, the ratio of $\sigma$ to the group delay imposed by the entire length of MCF equals $8.7 \cdot 10^{-5}$, which nevertheless testifies to a high homogeneity between cores. We conclude that the intrinsic inter-core GDD is random and normally distributed. At this point it is interesting to compare our results with [10], by Mosley et al., who used a different approach (based on intensity measurements and a Markov chain Monte Carlo process as a reconstruction algorithm) to map out the propagation constants in a 37-core fiber. Two discrepancies are immediately apparent: (1) we observe a higher relative standard deviation compared to the estimated width of the normalized distribution of the propagation constants (Fig. $\underline{4(\mathrm{~d})}$ in [10]), at least a factor of two, judging by eye; and (2) contrary to [10], we observe no systematic differences between the inner and outer cores-i.e., a sliding average of the data in Fig. 7(a) would yield the same mean value. Also, no bias is apparent from the histogram in Fig. 7(b). We have insufficient information to establish the origin of these discrepancies, but they might stem from differences in fabrication. Another interesting remark can be made here-as also pointed out in [10], by Mosley et al., the coupling between two cores is dependent on the difference in propagation constants between the two cores, being highest for zero difference. In MCF for lensless endsocopy low coupling is highly desirable, so intercore GDD could actually be seen as beneficial, and there may be an optimal trade-off between inter-core GDD and coupling between cores. 


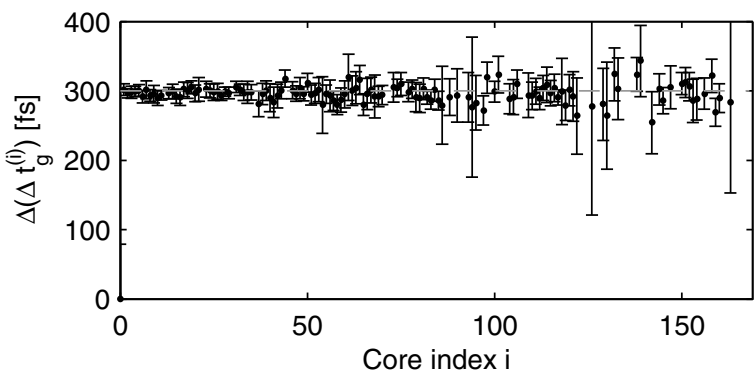

Fig. 8. Measured group delays with beamlets 1-168 experiencing $\delta t_{g}=300 \mathrm{fs}$ in the plane $\Sigma_{2}$. (Dots) Measured $\Delta\left(\Delta t_{g}^{(i)}\right)$, (gray dashed) expected delay from glass plate. Beamlets with $1 \leq i \leq 168$ pass through glass while the beamlet with $i=0$ passes through a hole (no glass). The group delays in Fig. 7(a) have been taken as baseline for the values presented in this Figure. Only 126 out of 168 values are shown. Error bars, three standard deviations.

It could be observed that the error bars in Fig. 7(a) appear to be larger with increasing core index. This occurs due to the power imbalance between the cores, which leads to a lower fringe contrast in the phase-shifting interferometry measurement. Hence, we might expect some of these $\Delta t_{g}^{(i)}$ values to be erroneous. In order to establish the validity of the $\Delta t_{g}^{(i)}$ measurement in Fig. 7(a), we experimentally impose an additional known delay $\delta t_{g}=300 \mathrm{fs}$ on all cores but the reference core $i=0$. Subsequently we redo the group delay measurement; the results are presented in Fig. $\underline{8}$ as $\Delta\left(\Delta t_{g}^{(i)}\right)$, meaning the current measurement with Fig. $7(\mathrm{a})$ is subtracted.

This now gives us two gauges of the measurement uncertainty: (1) the confidence interval from the fit (the error bars) and (2) the comparison with the known value of the imposed $\delta t_{g}$. This test allows us to distinguish good $\Delta t_{g}^{(i)}$ measurements from bad ones. We fix as a criterion that $\Delta\left(\Delta t_{g}^{(i)}\right)$ must be within \pm 50 fs of $\delta t_{g}$. Here, 126 out of 168 fulfill this criterion. The outstanding 42 are classified as bad values. Out of these, 10 are due to inactive or locked segments on the DM, while the remainder are caused by the core birefringence, which leads to an output polarization state almost orthogonal to that of the reference core. In addition Fig. 8 allows us to estimate the precision of the method. Judging from the error bars, the precision is on the order of tens of femtoseconds in the best of cases.

\section{B. Compensation of Group Delay}

We experimentally confirmed that our compensation scheme was able to impose any delay on an arbitrary beamlet. A compensation plate with optical path length as shown in the inset in Fig. 9 was inserted in the plane $\Sigma_{1}$ so that each beamlet could experience one of three delays $\delta t_{g}$. To demonstrate this we let one third of the beamlets traverse each optical path (beamlet $i$ traverses $\left.[i \bmod 3] \delta t_{g}\right)$ and measured the resulting $\Delta t_{g}^{(i)}$ with phase-shifting spectral interferometry. The result is shown in Fig. 9. Indeed we recover three groups of values lying along the three equidistant dotted lines that denote the expected values.

Next, we experimentally demonstrate optimal compensation of all $\Delta t_{g}^{(i)}$ simultaneously. To do so, we must choose the appropriate $\delta t_{g}$, which is different from the one used in the test measurements above. We note from Fig. $\underline{6}$ that

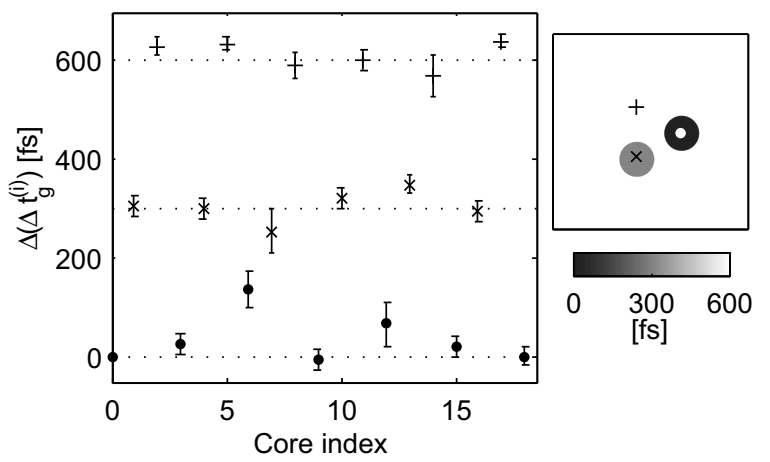

Fig. 9. Imposition of additional group delay on arbitrary cores. (Dots) Beamlets 0,3, ., 18 experience zero additional group delay. (Crosses) Beamlets 1, 4, .., 16 experience an additional group delay of $1 \cdot \delta t_{g}$. (Plusses) Beamlets 2,5, .., 17 experience an additional group delay of $2 \cdot \delta t_{g}$. Error bars, three standard deviations. Inset, sketch of the optical path length of the compensation plate used in the plane $\Sigma_{1}$.

coverslips of thickness $90 \mu \mathrm{m}$ are predicted to give the optimal compensation given a distribution slightly broader than the experimental one. As a compromise forced by the availability of coverslip thicknesses we choose a thickness of $100 \mu \mathrm{m}$ giving a compensation plate with $\delta t_{g}=170$ fs. Figure $7(\mathrm{c})$ shows the histogram of $\Delta t_{g}^{(i)}$ in the case in which the compensation scheme is active, as calculated from Fig. $7(\mathrm{~b})$ and Eq. (3) and $\delta t_{g}=170 \mathrm{fs}$. With the compensation scheme we thus expect to reduce the width of the distribution from 123 to $65 \mathrm{fs}$. We then thresholded the data in Fig. 7 to divide the beamlets into three groups destined to traverse different optical path lengths; this case is defined as the "compensated case" whereas the three cases in which all beamlets traverse the same optical path length are defined as the "uncompensated cases". In order to verify the performance gain using the compensated case we establish a focus $Z=1000 \mu \mathrm{m}$ from the distal tip of the MCF using a variant of the phase calibration algorithm we employed in [8] for all four cases. Figure 10 presents the resulting images of the focus acquired by CMOS1. Figures $10(\mathrm{a})$ and $10(\mathrm{~b})$ show the compensated case, and it can be appreciated that the focus intensity is $21 \%-37 \%$ higher than in Figs. $10(\mathrm{c})-10(\mathrm{~h})$, the uncompensated cases. In terms of energy in the focal spot, it, too, is higher by $22 \%-35 \%$. The setup was left untouched during the four measurements, which were commanded from a PC, which reinforces the conclusion that the increased intensity in the compensated case is really due to better temporal overlap of the composite beamlets, as expected.

\section{DISCUSSION}

We are fairly convinced that the concept of inter-core group delay compensation detailed in this report offers a superior trade-off between simplicity, flexibility, and efficiency: the simpler solution of inserting compensation plates not in the plane $\Sigma_{1}$ but in $\Sigma_{2}$ would likely have given similar end results but at the cost of highly elaborate compensation plates and a complete lack of flexibility as each MCF and each geometric configuration would require a specific compensation plate. We are also fairly convinced that our concept cannot be made to function 


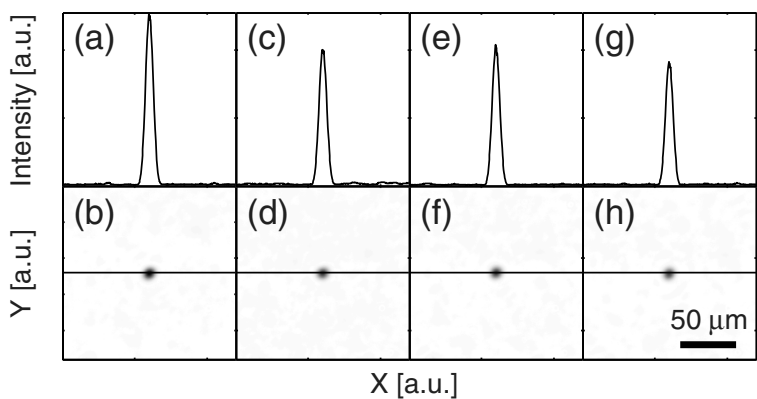

Fig. 10. Compensation of inter-core GDD. (Bottom row) Images of distal focus and (upper row) horizontal cuts. (a),(b) With group delay compensation, each beamlet passes through one of three points in the plane $\Sigma_{1}$. (c)-(h) Without group delay compensation, all beamlets pass through (c),(d) point $0,(\mathrm{e}),(\mathrm{f})$ point 1 , and $(\mathrm{g}),(\mathrm{h})$ point 2 in the plane $\Sigma_{1}$. With compensation the focus intensity increases by $21 \%-37 \%$ and the focus energy by $22 \%-35 \%$. The expected gain can be calculated from Eq. (2) and the $\Delta t_{g}^{(i)}$ data in Fig. 7. Doing so we find an expected gain of $47 \%$, not far from the measured one. The discrepancy might arise from imperfect spatial overlap of beamlets or differences in core birefringence, both leading to variation in intensity from beamlet to beamlet.

with less than two wavefront shapers (2D-SLM or DM). Any attempt to fold the setup and use two incidences on the same wavefront shaper would hit upon the fact that linear phaseconjugating mirrors do not exist. In order for a folded setup to work, a beamlet with $\left(x_{i}, y_{i}, k_{x, i}, k_{y, i}\right)$ emitted from a segment must come back to the same plane with $\left(x_{i}, y_{i},-k_{x, i}\right.$, $\left.-k_{y, i}\right)$. But with normal mirrors and telescopes, it can only come back to the same plane as $\left(x_{i}, y_{i}, k_{x, i}, k_{y, i}\right)$ or $\left(-x_{i},-y_{i},-k_{x, i}\right.$, $\left.-k_{y, i}\right)$. So folding is only really possible in one-beam settings in which $(x, y)=-(x, y)=(0,0)$. Please also note that in our concept there is no space-time coupling because the additional group delays are introduced in real space, not in momentum space, unlike in, for example, $4-f$ pulse shapers [14].

We have identified a few limiting factors at the present state, the first of which is the 7\% reflection loss at each pass through a coverslip. In the measurements above we had a maximum of two passes, which was not too much of a concern. In the case in which finer compensation of $\Delta t_{g}^{(i)}$ is desired, more plates would be needed and antireflection coatings become necessary in order to avoid excessive reflection losses. Applying the laser ablation detailed above also on coverslips with antireflection coating poses no fundamental problem. As an alternative, it might be possible to fabricate a one-block compensation plate by more advanced machining or etching techniques to decrease the number of reflective interfaces. Furthermore, in conjunction with reflective DMs and 2D-SLMs with very high light utilization efficiency (up to 95\%), this system would be compatible with the output powers of conventional laser sources already in use for multi-photon microscopes. Another limiting factor is the congestion of spots in the plane $\Sigma_{1}$. As can be gleaned from the inset in Fig. 2, the maximum number of different group delays that can be imposed on beamlets is given by the maximum number of nonoverlapping spots that can be generated in the plane $\Sigma_{1}$. In the present setup, this limit is set by the DM segment size and the maximum range of DM segment tip and tilt. In practice we found that in the present conditions four nonoverlapping spots were achievable. This allowed us to impose three different $\delta t_{g}$ on sets of beamlets, while the fourth setting $\Delta \vec{k}=(0,0)$ was used as a beam block. Also, larger DM segment size and a larger DM segment tip/tilt range would immediately lead to more degrees of freedom. For instance, existing DM technology already allows for a four times larger angular deflection leading immediately to an increase of $4 \times 4$ degrees of freedom for compensation. In the case of longer fiber lengths, the limiting factor would again relate to the range of compensation available. It is important to note that the inter-core GDD need not follow a simple linear accumulation of delay with length as extrinsic factors could partially compensate for this. Moreover for longer fibers, existing schemes for the compensation (for example, [15]) of chromatic dispersion can be directly applied to the laser beam before its division into beamlets as the core-to-core variation of the refractive indices $(\Delta n)$ is expected to be less than $10^{-4}$. Furthermore, it must be observed that we have not accounted for extrinsic factors, notably bending of the fiber on the intercore GDD. We expect this to arise from the stress-induced changes in the refractive index and to play a significant role. In the application of lensless endoscopes beyond a few special cases, an active compensation for bending is essential. This remains one of the key challenges toward the operation of robust lensless endoscopes in a clinical setting and represents the next logical step in the progression of our work. We would like to point out that our concept of compensation would allow for a fast compensation of the delay introduced by bending, provided it can be measured in real time $[16,17]$.

We must attach a few comments to our choice of using spectral interferometry rather than other methods in order to map out the $\Delta t_{g}^{(i)}$ of the MCF. Spectral interferometry necessitates no moving parts in the setup, contrary to time-domain methods, and from only a few spectra, it determines the relative delay of pulses up to a value limited only by the inverse of the spectrometer channel spacing. In addition, spectral interferometry with a fiber-coupled spectrum analyzer is potentially well-suited for in situ measurement of inter-core GDD of a lensless endoscope based on MCF (since this will change with the geometric configuration of the MCF).

We emphasize that the MCF subject to the present study was the same double-clad MCF as the one we used in our demonstration of two-photon endoscopic imaging [9]. The double cladding enables the backcollection of fluorescent signal, but it also leads to some parasitic background in the $\Delta t_{g}^{(i)}$ measurement because the inner clad guides any light impinging on the MCF proximal endface to the distal endface. However, as the above results show, this is a surmountable difficulty.

In the near future, advances in the design and fabrication of MCF, for example, MCF with a large number of cores with aligned birefringence [18], are expected to bring about significant performance gains in lensless endoscopy including the group delay compensation scheme described in this paper.

\section{CONCLUSION}

While gains in performance were modest in this report, we believe that we have presented a concept to measure and 
compensate for residual inter-core GDD and that it will eventually enable a two-photon lensless endoscope based on MCF in practical settings, as long as the endoscope probe can remain static. In particular a DM with larger tip/tilt range would immediately lead to a decrease of congestion in the plane $\Sigma_{1}$, and the compensation plates made up of larger numbers of coverslips can be easily machined, resulting in finer compensation of $\Delta t_{g}^{(i)}$. While endoscopic imaging was not the aim of this report, all the concepts presented here are compatible with two-photon lensless endoscopic imaging.

\section{APPENDIX A: PHASE-SHIFTING SPECTRAL INTERFEROMETRY}

\section{Collinear Case}

We use phase-shifting spectral interferometry to measure the inter-core GDD, meaning the relative group delays $\Delta t_{g}^{(i)}$ relative to core 0 of all $N$ beamlets at the distal end of the MCF.

The measurement is performed two cores at a time, one of which with $i=0$ serves as reference

$$
E^{(0)}(t)=\mathcal{E}(t)
$$

while the field of beamlet $i$ with group delay $\Delta t_{g}^{(i)}$ and phase $\phi^{(i)}$ is assumed to be of the form

$$
E^{(i)}(t)=\mathcal{E}\left(t-\Delta t_{g}^{(i)}\right) \mathrm{e}^{i \phi^{(i)}},
$$

which in the spectral domain is

$$
\tilde{E}^{(i)}(\omega)=\tilde{\mathcal{E}}(\omega) \mathrm{e}^{i \Delta t_{g}^{(i)} \omega} \mathrm{e}^{i \phi^{(i)}} .
$$

If two beamlets overlap collinearly, then the resulting spectrum is

$$
\begin{aligned}
\left|\tilde{E}^{(i)}(\omega)+\tilde{E}^{(0)}(\omega)\right|^{2} & =2|\tilde{\mathcal{E}}|^{2}\left[1+\cos \left(\Delta t_{g}^{(i)} \omega+\phi^{(i)}\right)\right] \\
& =2|\tilde{\mathcal{E}}|^{2}\left[1+\cos \left(\Psi^{(i)}(\omega)\right)\right] .
\end{aligned}
$$

This expression remains valid in the case in which both beamlets are not transform-limited as long as the chirp is common to both. Since all beamlets all go through the same optical elements or near-identical cores, we expect this to be the case. From this we see that the relative group delay comes about as the derivative with respect to $\omega$ of the spectral phase $\Psi^{(i)}(\omega)$.

We now describe our experimental approach to measure $\Psi^{(i)}$ in a robust way. We consider fixed $\omega$ (experimentally, one spectral channel of the OSA onto which the two beamlets are incident). We introduce an additional phase $\phi_{j}$ on beamlet $i$. Experimentally, $\phi_{j}$ is set by the piston of DM segment $i$. And during the measurement all beamlets but beamlets $i$ and 0 are blocked by a spatial filter in $\Sigma_{1}$; the DM imposes a large positive transverse $k$ vector on beamlets $i$ and 0 and a large negative transverse $k$ vector on all the rest, which assures good extinction in $\Sigma_{1}$. We acquire spectra $S^{(i)}$ for four equidistant $\phi_{j}=j \frac{3 \pi}{2}$, $0 \leq j \leq 3$. The spectra $S^{(i)}\left(\phi_{j}\right)$ thus measured are

$$
S^{(i)}\left(\phi_{j}\right)=2|\tilde{\mathcal{E}}|^{2}\left[1+\cos \left(\Psi^{(i)}(\omega)+\phi_{j}\right)\right]
$$

From this stack of spectra we can extract the spectral phase $\Psi^{(i)}(\omega)$ as [19]

$$
\Psi^{(i)}(\omega)=-\arctan \frac{\sum_{j=0}^{3} S^{(i)}\left(\phi_{j}\right) \sin \left(\phi_{j}\right)}{\sum_{j=0}^{3} S^{(i)}\left(\phi_{j}\right) \cos \left(\phi_{j}\right)} .
$$

This method is robust toward effects such as material dispersion, spectral speckle, and polarization mode dispersion in the multimode probe of the OSA (as long as the probe remains static for the measurement duration). This is repeated for all the channels of the OSA over three pulse spectral bandwidths FWHM, centered on the spectral maximum. Subsequently, the group delay that we were searching for can be found as

$$
\Delta t_{g}^{(i)}=\frac{\mathrm{d} \Psi^{(i)}(\omega)}{\mathrm{d} \omega} .
$$

However in practice, we extract $\Delta t_{g}^{(i)}$ from a linear fit to $\Psi^{(i)}(\omega)$.

\section{Noncollinear Case}

Experimentally, the interfering beamlets are not collinear on the probe of the OSA. The measurement is performed in the plane CMOS2 in Fig. 2. In this case we can assume the field of the reference beamlet $E^{(0)}$ to be as before, and the field of beamlet $i$ to be of the form

$$
E^{(i)}(t)=\mathcal{E}\left(t-\Delta t_{g}^{(i)}\right) \mathrm{e}^{i k_{x}^{(i)}} \mathrm{e}^{i \phi^{(i)}},
$$

where $k_{x}^{(i)}$ is the transverse $k$ vector. In the spectral domain this gives

$$
\tilde{E}^{(i)}(\omega)=\tilde{\mathcal{E}}(\omega) \mathrm{e}^{i \Delta t_{g}^{(i)} \omega} \mathrm{e}^{i k_{x}^{(i)}+i k z} \mathrm{e}^{i \phi^{(i)}} .
$$

And the spectrum arising from the interference between the two beamlets 0 and $i$ is

$$
\begin{aligned}
& \int_{-D / 2}^{D / 2} \mathrm{~d} x\left|\tilde{E}^{(i)}(\omega)+\tilde{E}^{(0)}(\omega)\right|^{2} \\
& \quad=2|\tilde{\mathcal{E}}|^{2} \int_{-D / 2}^{D / 2} \mathrm{~d} x\left[1+\cos \left(\Psi^{(i)}(\omega)+k_{x}^{(i)} x\right)\right],
\end{aligned}
$$

where $D$ is the diameter of the probe of the OSA. We see that in the case $k_{x}^{(i)} D \ll 2 \pi$ we recover the collinear case, up to a phase offset that disappears upon taking the derivative Eq. (A7). However, if $k_{x}^{(i)} D \gg 2 \pi$ the spectral fringes will average out and the measurement of $\Delta t_{g}^{(i)}$ will fail. With the parameters in our setup, $k_{x}^{(i)} D<2.7$, which remains within the acceptable range. It is possible as well to perform the measurement by placing the OSA probe not in a Fourier plane of the MCF endface but at a distance $Z$ from the endface. In that case with the current MCF parameters the criterion becomes $Z>5 \mathrm{~mm}$.

Centre Nationale de la Recherche Scientifique (CNRS) interdisciplinary mission; Contrats de Projets État-Région Campus Intelligence Ambiante (CPER-CIA 2007-2013); French National Research Agency (L' Agence Nationale de la Recherche) (ANR-10-INSB-04-01, ANR-11-EQPX-0017, ANR-11-IDEX-0001-02, ANR-11-INSB-0006, ANR-11LABX-0007, ANR-14-CE17-0004); Fondation pour la Recherche Médicale (FRM) (DBS20131128448); Nord-Pas de Calais Regional Council and European Regional Development Fund (ERDF); Weizmann $\mathrm{NaBi}$ European Associated Laboratory.

G. B. thanks K. Delplace and D. Labat for providing technical support. 


\section{REFERENCES AND NOTES}

1. A. P. Mosk, A. Lagendijk, G. Lerosey, and M. Fink, "Controlling waves in space and time for imaging and focusing in complex media," Nat. Photonics 6, 283-292 (2012).

2. Y. Choi, C. Yoon, M. Kim, T. D. Yang, C. Fang-Yen, R. R. Dasari, K. J. Lee, and W. Choi, "Scanner-free and wide-field endoscopic imaging by using a single multimode optical fiber," Phys. Rev. Lett. 109, 203901 (2012).

3. T. Cizmar and K. Dholakia, "Exploiting multimode waveguides for pure fibre-based imaging," Nat. Commun. 3, 1027 (2012).

4. I. N. Papadopoulos, S. Farahi, C. Moser, and D. Psaltis, "Highresolution, lensless endoscope based on digital scanning through a multimode optical fiber," Biomed. Opt. Express 4, 260-270 (2013).

5. S. Bianchi, V. P. Rajamanickam, L. Ferrara, E. Di Fabrizio, C. Liberale, and R. Di Leonardo, "Focusing and imaging with increased numerical apertures through multimode fibers with micro-fabricated optics," Opt. Lett. 38, 4935-4938 (2013).

6. E. E. Morales-Delgado, S. Farahi, I. N. Papadopoulos, D. Psaltis, and C. Moser, "Delivery of focused short pulses through a multimode fiber," Opt. Express 23, 9109-9120 (2015).

7. A. J. Thompson, C. Paterson, M. A. A. Neil, C. Dunsby, and P. M. W. French, "Adaptive phase compensation for ultracompact laser scanning endomicroscopy," Opt. Lett. 36, 1707-1709 (2011).

8. E. R. Andresen, G. Bouwmans, S. Monneret, and H. Rigneault, "Toward endoscopes with no distal optics: video-rate scanning microscopy through a fiber bundle," Opt. Lett. 38, 609-611 (2013).

9. E. R. Andresen, G. Bouwmans, S. Monneret, and H. Rigneault, "Two-photon lensless endoscope," Opt. Express 21, 20713-20721 (2013).

10. P. J. Mosley, I. Gris-Sanchez, J. M. Stone, R. J. A. Francis-Jones, D. J. Ashton, and T. A. Birks, "Characterizing the variation of propagation constants in multicore fiber," Opt. Express 22, 25689-25699 (2014).

11. P. S. J. Russell, "Photonic-crystal fibers," J. Lightwave Technol. 24, 4729-4749 (2006).

12. The number of segments of the DM (163) is less than the number of cores of the MCF (169). The DM is missing the six segments corresponding to MCF core indices 127, 134, 141, 148, 155, and 162. Therefore, no group delay compensation can be exerted on these six cores in our compensation scheme. For completeness we have, however, measured the $\Delta t_{g}^{(i)}$ of these six cores, but using the 2D-SLM rather than the DM to effectuate the phase-shifting spectral interferometry. These six values are present in Fig. 7 but not in any of the subsequent figures.

13. L. Lepetit, G. Cheriaux, and M. Joffre, "Linear techniques of phase measurement by femtosecond spectral interferometry for applications in spectroscopy," J. Opt. Soc. Am. B 12, 2467-2474 (1995).

14. A. M. Weiner, "Femtosecond pulse shaping using spatial light modulators," Rev. Sci. Instrum. 71, 1929-1960 (2000).

15. R. L. Fork, O. E. Martinez, and J. P. Gordon, "Negative dispersion using pairs of prisms," Opt. Lett. 9, 150-152 (1984).

16. A. M. Caravaca-Aguirre, E. Niv, D. B. Conkey, and R. Piestun, "Real-time resilient focusing through a bending multimode fiber," Opt. Express 21, 12881-12887 (2013).

17. S. Farahi, D. Ziegler, I. N. Papadopoulos, D. Psaltis, and C. Moser, "Dynamic bending compensation while focusing through a multimode fiber," Opt. Express 21, 22504-22514 (2013).

18. J. M. Stone, F. Yu, and J. C. Knight, "Highly birefringent 98-core fiber," Opt. Lett. 39, 4568-4570 (2014).

19. J. H. Bruning, D. R. Herriott, J. E. Gallagher, D. P. Rosenfeld, A. D. White, and D. J. Brangaccio, "Digital wavefront measuring interferometer for testing optical surfaces and lenses," Appl. Opt. 13, 2693-2703 (1974). 